\title{
Incidental MDCT finding of a subclinical PDA in an adult patient with Eisenmenger syndrome
}

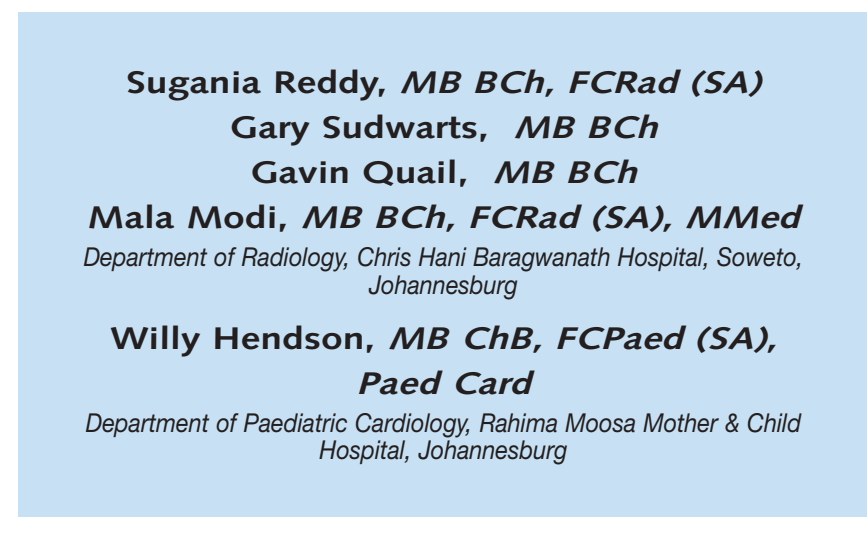

\section{Introduction}

Patent ductus arteriosus (PDA) comprises $5-10 \%$ of all congenital cardiac lesions. ${ }^{1}$ The incidence of PDA is 1 in 500, although most of these children are asymptomatic. ${ }^{2}$ The defect must persist beyond 3 months of age to be considered pathological. ${ }^{1}$ The male:female ratio is 1:2 unless the condition is associated with other congenital problems. ${ }^{3}$ Most children with PDA are asymptomatic; however, $50 \%$ of patients with a large PDA will develop pulmonary hypertension ${ }^{4}$ and may eventually develop Eisenmenger's syndrome if left patent.

Eisenmenger's syndrome is an irreversible condition where there is a reversal of flow of a congenital systemic to pulmonary shunt that occurs in untreated congenital cardiac defects. A left-to-right shunt is converted into a right-to-left shunt secondary to the elevated pulmonary artery pressures and associated pulmonary vascular disease.

\section{Clinical findings}

We describe the case of a 23-year-old woman who presented with acute abdominal pain and shortness of breath. Her history revealed positive HIV status with a CD4 count of $39 \times 10^{\%} / \mathrm{ml}$. On clinical examination, there was a palpable $\mathrm{p} 2$ and clinically suspected pulmonary arterial hypertension. There was no differential clubbing and no machinery murmur at the left sternal border. The patient had a history of longstanding mitral regurgitation.

It is well known that large PDAs may have no audible murmur. Once Eisenmenger's syndrome is established, the murmur may disappear owing to the right- and left-side pressures being equal. ${ }^{4}$

The patient underwent emergency laparotomy for the abdominal pain. An ectopic fetus was removed but she continued to saturate

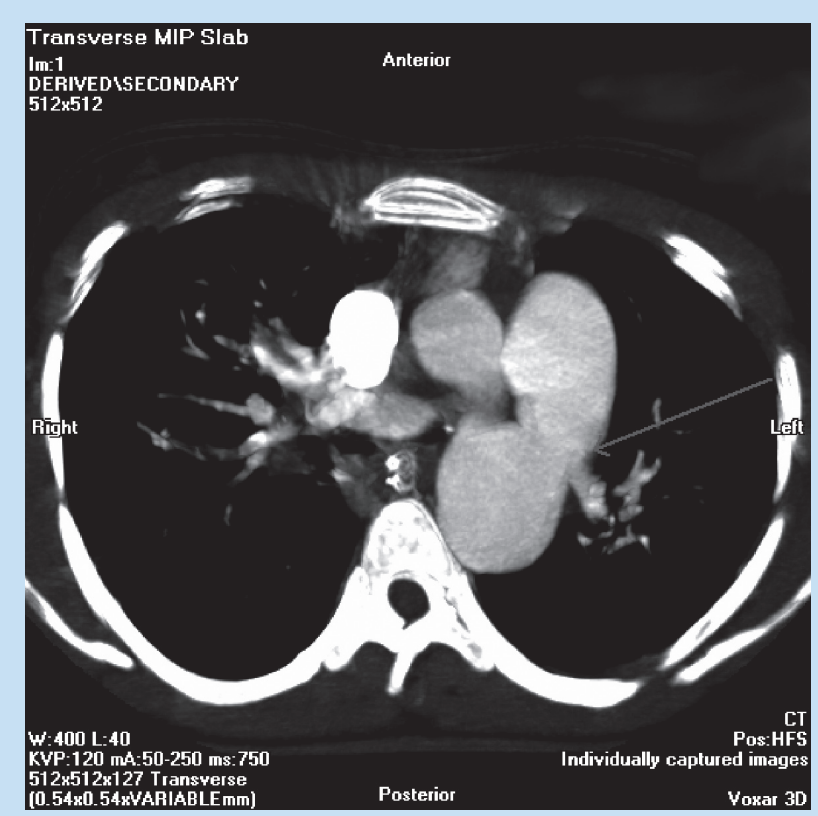

Fig. 1. Multislice spiral pulmonary angiogram: Axial maximum intensity projection; arrow points to PDA.

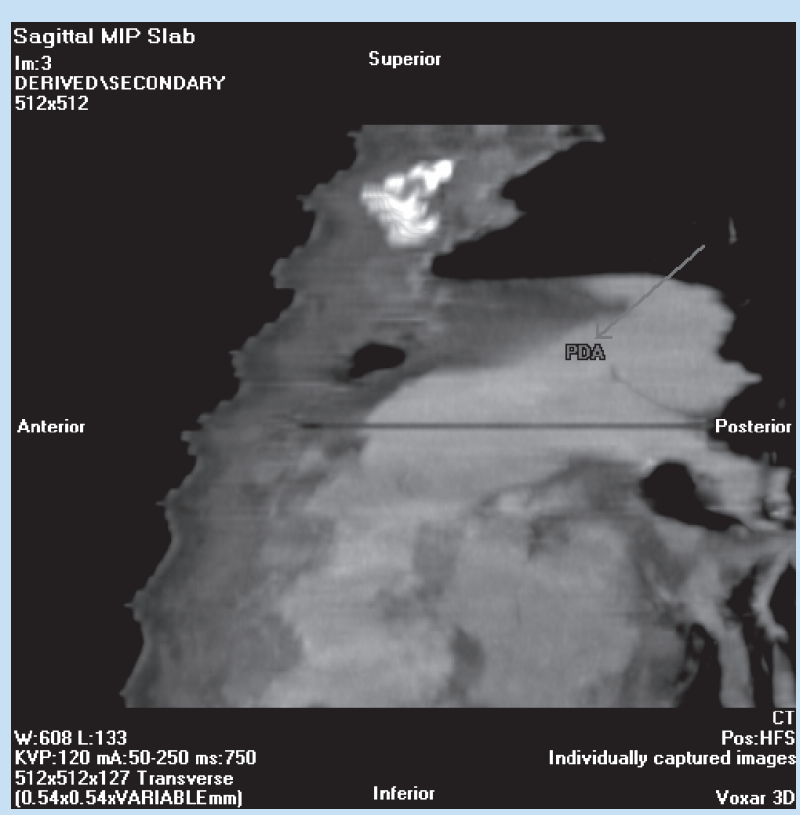

Fig. 2. Multislice spiral pulmonary angiogram: Sagittal maximum intensity projection; arrow points to PDA. 


\section{CASE REPORT}

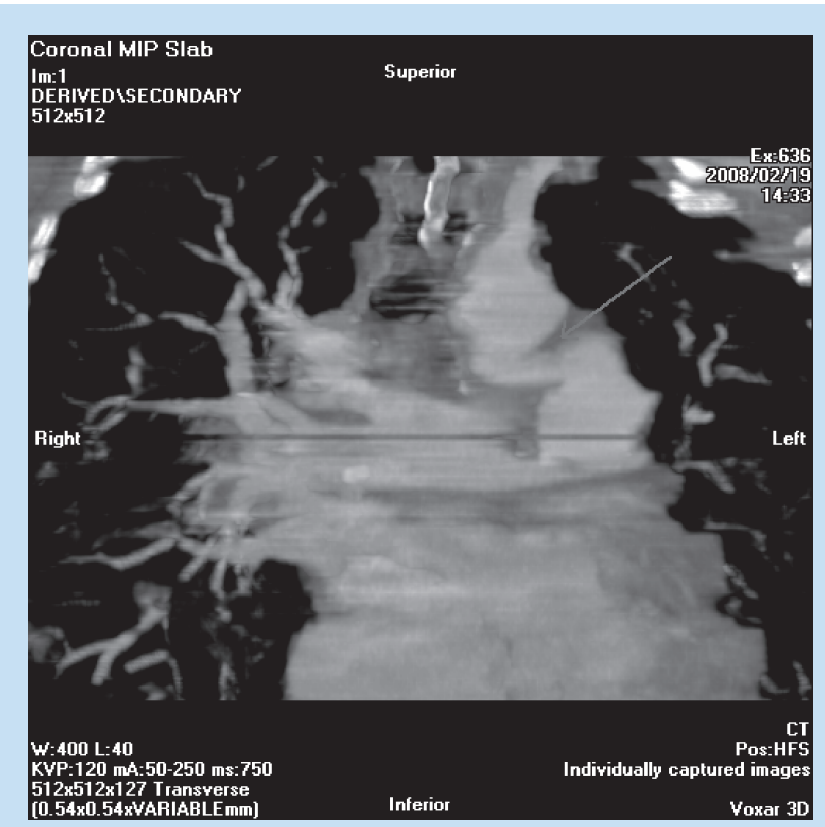

Fig. 3. Multislice spiral pulmonary angiogram: Coronal maximum intensity projection; arrow points to $P D A$.

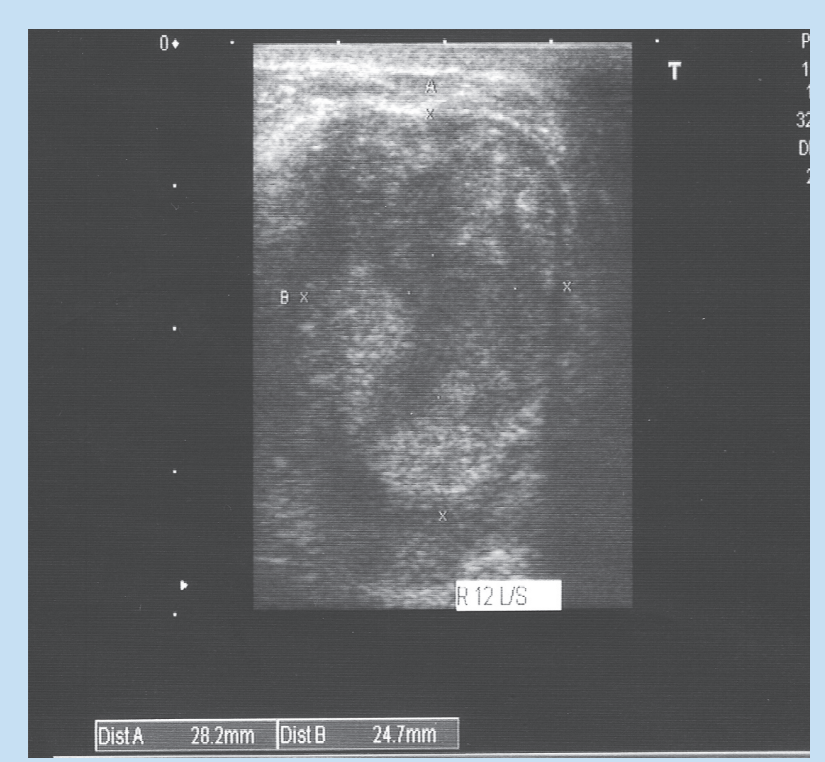

Fig. 4. Chest radiograph: Enlarged cardiac shadow shows enlarged right atrium and ventricle. Filling in of the aorto-pulmonary window is suggestive of pulmonary hypertension. Patchy consolidation of the right lower lobe.

poorly; however, no differential peripheral arterial saturation was recorded. Post surgery, she was deemed not extubatable by the attending anaesthetist.

The initial postoperative arterial blood gas on $100 \%$ oxygen showed severe respiratory acidosis and hypercapniea with a $\mathrm{pH}$ of 7.05, $\mathrm{PCO}_{2}$ of $80 \mathrm{mmHg}$ and $\mathrm{PaO}_{2}$ of $66 \mathrm{mmHg}$ and $\mathrm{BE}$ of -10.5 . Haemoglobin was $10.8 \mathrm{~g} / \mathrm{dl}$ and haematocrit $35 \%$. A portable transthoracic echo was performed and showed an ejection fraction

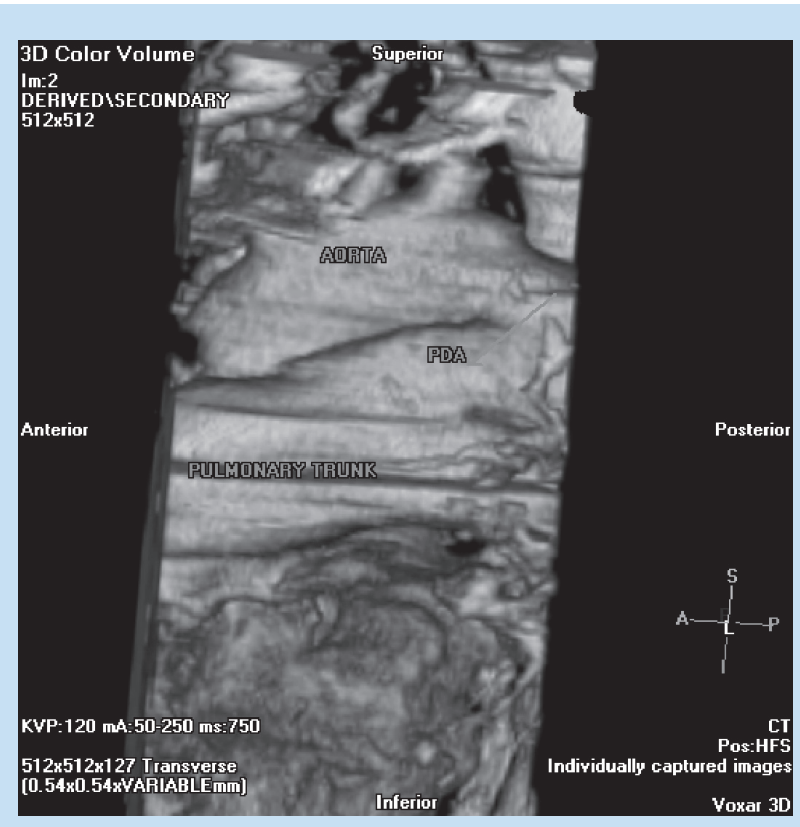

Fig. 5. Multislice spiral pulmonary angiogram: $3 D$ reconstruction, sagittal image; arrow points to PDA.

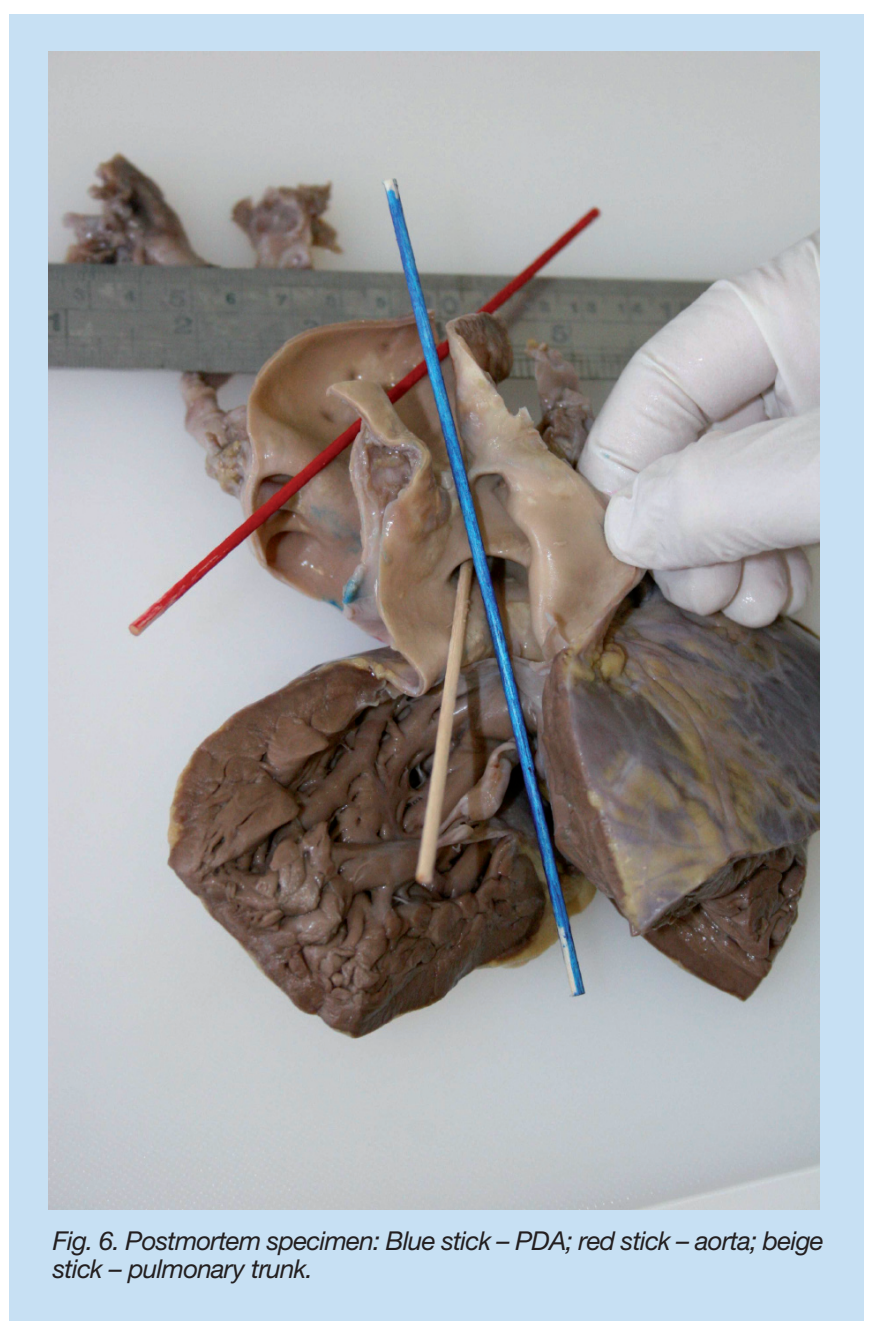




\section{CASE REPORT}

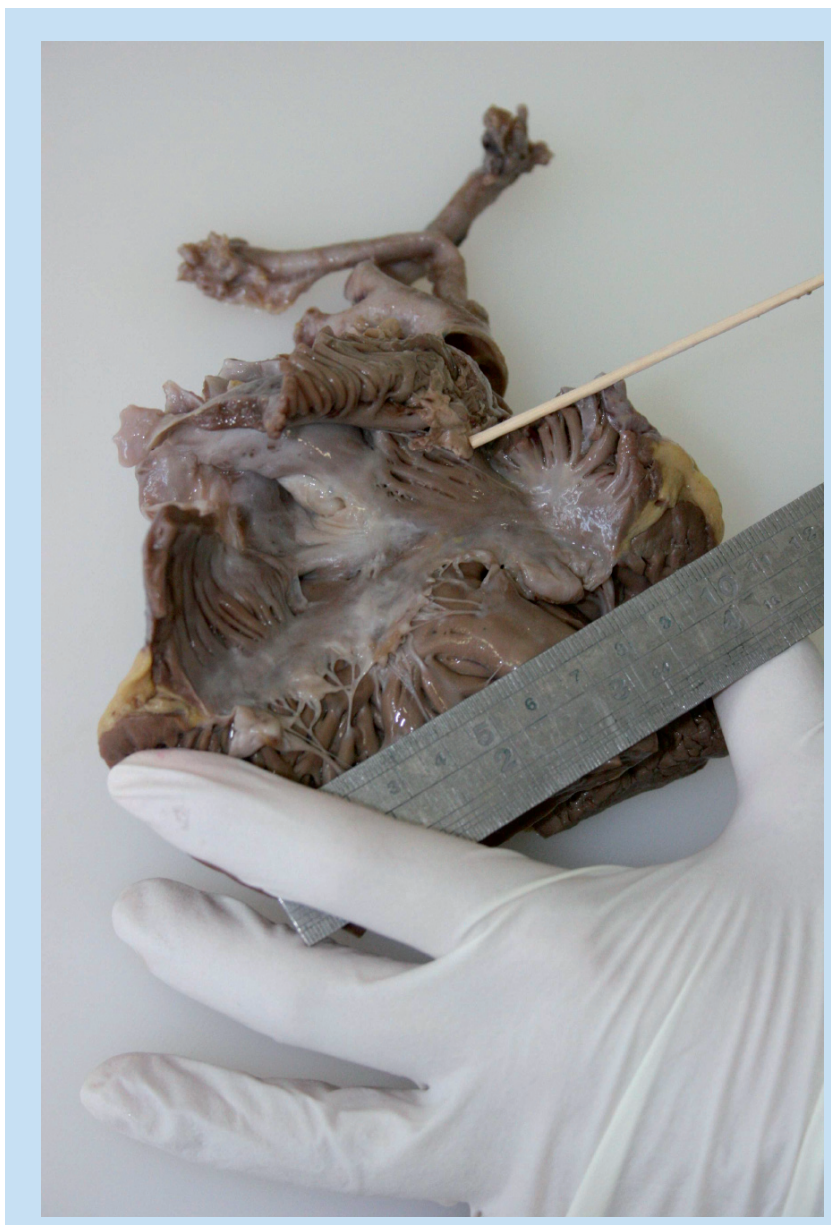

Fig. 7. Postmortem specimen showing thrombus in right ventricle.

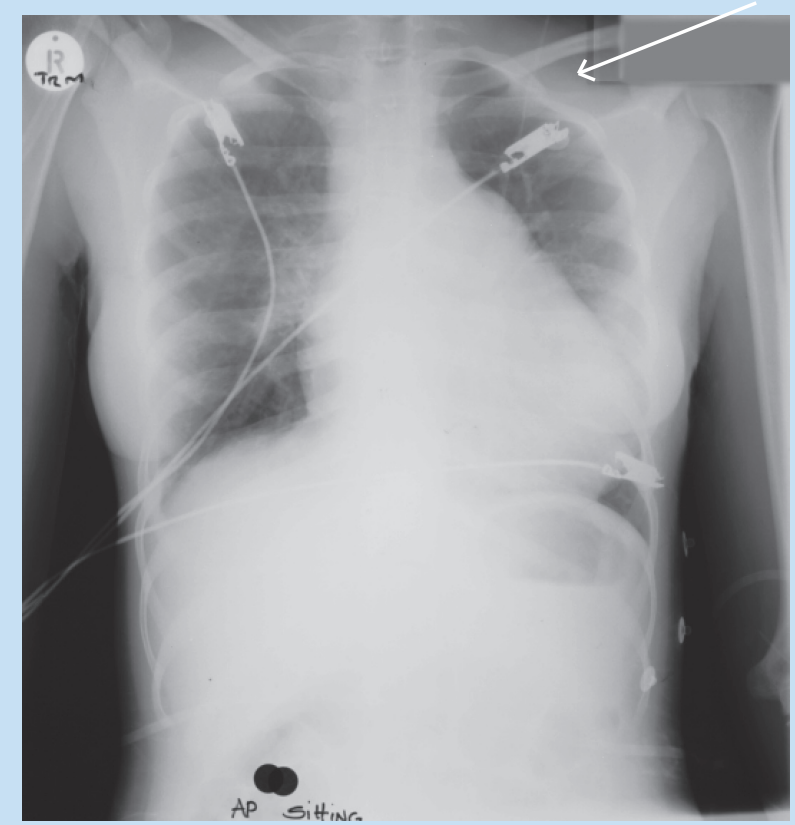

Fig. 8. Chest $X$-ray image. of $60 \%$ with a normal mitral valve. There was also triscupid regurgitation, a dilated right ventricle and pulmonary arterial hypertension.

A pulmonary embolus was suspected as the likely cause of the pulmonary hypertension. A computed tomographic (CT) angiogram of the patient's chest was arranged urgently and the surprising presence of a large PDA was found.

Findings on multidetector-row CT (MDCT) in ow patient (Figs 1-3) indicated:

- presence of PDA (12 mm diameter)

- presence of pulmonary hypertension; main pulmonary trunk $>3 \mathrm{~cm}$

- presence of enlarged RV and RA

- thrombus present in the RA; subsegmental pulmonary emboli.

Despite initial improvement, our patient deteriorated and died in the ICU 6 days post laparotomy. A postmortem examination was performed and revealed:

- 10 mm diameter PDA

- histological evidence of pulmonary hypertension characterised by myointimal proliferation and muscular hypertrophy

- multiple small pulmonary thrombo-emboli

- right-sided bronchopneumonia

- histological features of diffuse alveolar damage.

\section{Imaging}

\section{Chest radiography}

The features on the chest radiograph most suggestive of a PDA complicated by Eisenmenger's syndrome are:

- filling in of the aorto-pulmonary window and enlargement of the pulmonary arteries

- pruning of the pulmonary arteries peripherally

- cardiomegaly, especially right-sided enlargement

- left atrium and left ventricle can eventually return to normal size as the left-to-right shunt reduces with elevated pulmonary pressures

- calcification of the PDA in elderly patients is sometimes visible, especially on a lateral chest radiograph.

The above findings are unfortunately nonspecific and are usually only appreciated in retrospect. Any cause of a left-to-right shunt can give similar features (e.g. endocardial cushion defects). The most reliable (but uncommon) sign is a calcified PDA 'railroad track' sign. $^{5-8}$

In our patient, Fig. 4 demonstrates an enlarged cardiac shadow with an enlarged right atrium and right ventricle. The aortopulmonary window is filled in, and there is peripheral pruning suggestive of pulmonary hypertension. There is also patchy consolidation of the right lower lobe.

\section{Echo findings}

Typical findings are left atrial enlargement exceeding aortic root diameter and colour flow which usually shows reverse pulmonary artery flow in diastole. 
A trans-oesophageal echocadiogram is superior to a transthoracic echocardiogram (TTE) in adolescents and adults for the diagnosis. ${ }^{9}$ However, TTE is far more commonly used in diagnosing PDAs as it is less invasive. Once Eisenmenger's syndrome is established, the echo will reveal pulmonary hypertension but may miss the PDA (owing to the reversed flow across the shunt). ${ }^{1}$ Our patient underwent TTE. Pulmonary hypertension was diagnosed. She was found to have an enlarged right ventricle and tricuspid regurgitation (decompensated right heart because of Eisenmenger's syndrome). The PDA was not identified.

\section{Angiography}

CT angiography (CTA) and magnetic resonance angiography (MRA) have largely replaced formal angiography in the investigation of PDAs as the former modalities are less invasive and allow imaging of related anatomy and complications.

Excellent images can be obtained with formal angiography. The PDA will opacify well from the presence of relatively high concentrations of intra-arterial contrast media being injected into the ascending aorta (or even in a pulmonary angiogram, especially if Eisenmenger's syndrome is established). Dimensions of the PDA are then easily measured.

Once Eisenmenger's syndrome (i.e. shunt reversal) is established, the PDA may not be visible on the aortic runs. In such a situation (or in young patients in whom venous punctures are preferable), the femoral vein can be cannulated and a catheter passed into the right side of the heart and into the pulmonary artery and through the PDA ${ }^{5,6}$ If Eisenmenger's is suspected, cardiac catheterisation is mandatory to measure the pulmonary resistance and thus assess for operability.

Clearly, angiography must be performed if percutanous intervention has been chosen as the course of management. In such cases, it may be useful to temporarily occlude the PDA and assess the resultant haemodynamic changes.

\section{Multidetector computed tomography (MDCT)}

MDCT has become an important investigation in patients with PDA, especially those with associated pulmonary hypertension and Eisenmenger's syndrome. ${ }^{10}$ Typical findings on MDCT of an uncomplicated PDA would include a tubular structure connecting the descending aorta with the distal main pulmonary artery or the proximal left pulmonary artery. Accurate measurements including narrowest and widest diameter of the PDA as well as length of the PDA can accurately and easily be done on MDCT to assist surgical management. ${ }^{11}$ MDCT also demonstrates the extent of calcification associated with the PDA, which will influence surgical management. ${ }^{12}$

MDCT assists in evaluating the direction of the shunt associated with a PDA. Studies timed for the pulmonary artery will show a 'negative jet' of unenhanced blood from the aorta to the pulmonary artery via the PDA. Studies timed for the aorta will show a 'positive jet' of enhanced blood flowing from the aorta to the unenhanced pulmonary artery via the PDA. ${ }^{11}$
In patients with PDAs complicated by Eisenmenger's syndrome, MDCT is more sensitive than transthoracic cardiac echo in detecting a PDA; this is because of the usual lack of the typical colour flow associated with a PDA when the PDA is complicated by Eisenmenger's syndrome (i.e. the expected direction of flow is reversed).$^{10}$ MDCT helps to differentiate mimickers of PDA such as ductus bump (remnant of the ductus arteriosus), aortic dissection or aortic aneurysm. One can also exclude PDAassociated complications on MDCT such as cardiomegaly (LV and LA involvement), pulmonary hypertension (pulmonary artery $>3$ $\mathrm{cm}$ ), Eisenmenger's syndrome (shunt reversal, enlarged RV +/- RA), pulmonary oedema, pulmonary infection, pulmonary emboli, and the effects of infective endocarditis.

\section{Magnetic resonance imaging and magnetic resonance angiography}

Magnetic resonance imaging (MRI) and magnetic resonance angiography (MRA) can demonstrate PDA complications such as pulmonary hypertension and ventricular dilatation with associated valvular regurgitation. ${ }^{13}$ Cine cardiac MRI assists in differentiating uncomplicated PDAs (low signal intensity in main pulmonary artery and descending aorta) from PDAs with Eisenmenger's syndrome (low signal intensity but reversal of flow). ${ }^{14}$

\section{Conclusion}

PDAs complicated by Eisenmenger's syndrome are notoriously difficult to detect on transthoracic echocardiography. The surprise finding in our patient of a large PDA on MDCT confirms the superior sensitivity of MDCT compared with transthoracic echocardiography in patients with Eisenmenger's syndrome.

We thank Dr L Blackburn and Professor M J Hale of the Department of Anatomical Pathology, Chris Hani Baragwanth Hospital, for completing the postmortem examination.

1. Schneider DJ, Moore JW. Patent ductus arteriosus. Circulation 2006; 114: 1873-1882.

2. Lloyd TR, Beekman RH III. Clinically silent patent ductus arteriosus. Am Heart J 1994; 127: 1664-1665. Letter.

3. Neish SR. Patent Ductus Arteriosus. emedicine, 2006. http://www.emedicine.com/PED/topic1747.htm (accessed 2 September 2008).

4. El-Chami MF. Eisenmenger Syndrome. emedicine, 2008. http://www.emedicine.com/med/TOPIC642. HTM (accessed 27 August 2008).

5. Hartnell GG. Patent Ductus Arteriosus. emedicine, 2007. http://www.emedicine.com/radio/TOPIC532 HTM (accessed 2 December 2008)

6. Sutton D. Textbook of Radiology and Imaging. 7th ed. St. Louis, MO, USA: Churchill Livingstone, 2007.

7. Dähnert W. Radiology Review Manual. 6th ed. Philadelphia, PA, USA: Lippincott Williams \& Wilkins, 2007.

8. Myers JL. Pulmonary Hypertension, Eisenmenger Syndrome. emedicine, 2007. http://www.emedicine. com/ped/topic2528.htm (accessed 2 December 2008).

9. Shyu KG, Lai LP, Lin SC, Chang H, Chen JJ. Diagnostic accuracy of transesophageal echocardiography for detecting patent ductus arteriosus in adolescents and adults. Chest 1995; 108(5): 1201-1205.

10. Yilmaz R, Demirbag R. The diagnosis of patent ductus arteriosus with Eisenmenger's syndrome by threedimensional computed tomography: a case report. Int J Cardiol 2005; 105(1): 96-97.

11. Goitein O, Fuhrman CR, Lacomis JM. Incidental finding on MDCT of patent ductus arteriosus: use of CT and MRI to assess clinical importance. Am J Roentgenol 2005; 184(6): 1924-1931.

12. Morgan-Hughes GJ, Marshall AJ, Roobottom C. Morphologic assessment of patent ductus arteriosus in adults using retrospectively ECG-gated multidetector CT. Am J Roentgenol 2003; 181(3): 749-754

13. Gaba RC, Carlos RC, Weadock WJ, Reddy GP, Sneider MB, Cascade PN. Cardiovascular MR imaging technique optimization and detection of disease in clinical practice. Radiographics 2002; 22(6): e6.

14. Hartnell GG, Cohen MC, Meier RA, Finn JP. Magnetic resonance angiography demonstration of congenital heart disease in adults. Clin Radiol 1996; 51(12): 851-857. 